\title{
Extracorporeal membrane oxygenation for the support of pediatric patients with acute fulminant myocarditis
}

\author{
Güntülü Şık ${ }^{1}$, Agageldi Annayev ${ }^{1}$, Asuman Demirbuğa ${ }^{1}$, Elif Deliceo ${ }^{1}$, Selim Aydın², \\ Ersin Erek ${ }^{2}$, Halil İbrahim Demir ${ }^{3}$, Agop Çıtak ${ }^{1}$ \\ Departments of ${ }^{1}$ Pediatric Intensive Care Unit, ${ }^{2}$ Cardiovascular Surgery, and ${ }^{3}$ Pediatric Cardiology, Acıbadem Mehmet Ali \\ Aydinlar University, School of Medicine, Istanbul, Turkey.E-mail: drguntulu@hotmail.com \\ Received: 25th June 2018, Revised: 31st August 2018, Accepted: 19th October 2018
}

\begin{abstract}
SUMMARY: Şık G, Annayev A, Demirbuğa A, Deliceo E, Aydın S, Erek E, Demir HI, Çıtak A. Extracorporeal membrane oxygenation for the support of pediatric patients with acute fulminant myocarditis. Turk J Pediatr 2019; 61: 867-872.
\end{abstract}

Acute fulminant myocarditis, is a severe, rapidly progressive disease. The clinical outcomes of children with severe acute myocarditis who are resist to medical treatment is not well known. We studied the clinical courses of patients with acute fulminant myocarditis supported by extracorporeal membrane oxygenation (ECMO). We performed a retrospective chart review of six children with acute fulminant myocarditis who were treated with ECMO. Demographic information, clinical and vital signs, as well as laboratory results were investigated. The median age of 63 months (13-140 months), the mean ECMO duration was 164 hours (79-402 hours), and median intensive care unit stay was 24 days. The most common symptoms were chest pain (66\%) and fever $(66 \%)$. Severe arrhythmia were seen in two patients. One patient received extracorporeal cardiopulmonary resuscitation. In two patients, right femoral arteries and right femoral veins were used, in others, right common carotid artery and right internal jugular veins were used. Five patients (83.3 \%) survived to discharge. ECMO can be used effectively in pediatric patients with acute fulminant myocarditis to support the circulation while awaiting myocardial recovery. Timely use of ECMO can improve the survival rate and may be associated with better outcomes.

Key words: acute fulminant myocarditis, extracorporeal membrane oxygenation, pediatric intensive care.

Acute fulminant myocarditis (AFM), is a severe, rapidly progressive disease resulting from cardiac muscle inflammation. Although most commonly due to viral infections, it can be seen as a result of other infections, autoimmune diseases, and intoxications. Among the viral agents most commonly encountered are enteroviruses, influenza, parvovirus 19, Epstein-Barr virus (EBV), human herpes virus 6 (HHV6) and cytomegalovirus (CMV)., ${ }^{1,2}$ The clinical presentation can extend from common cold to severe heart failure. Most common symptoms are nausea, vomiting, fever, abdominal pain, altered consciousness, dysrhythmias and respiratory distress. ${ }^{3}$ In acute myocarditis, supportive treatment, inotropic drugs, and mechanical ventilation can be sufficient for patients with mild disease, but for patients with severe fulminant myocarditis showing resistance to medical treatment, extracorporeal support techniques are lifesaving. ${ }^{4,5}$ The survival ratio in extracorporeal support techniques is $50-80 \% .^{6,7}$

In this study, our purpose was to establish the clinical features and short-term results of patients admitted to our center with a diagnosis of AFM resistant to medical 
treatment who received extracorporeal membrane oxygenation (ECMO).

\section{Material and Methods}

Six patients who were admitted to Acibadem University Pediatric Intensive Care Unit (PICU) between 2015 and 2017 who received ECMO due to lack of response to medical treatment were evaluated. Three patients were male, 3 patients were female, and their mean age was 63 months (range, 13-140 months). The mean ECMO duration was 164 hours, and the mean length of intensive care stay was 24 days.

Ethical approval was obtained from the Ethics Committee of Acibadem University, Faculty of Medicine (2018-9/23) and consent was given by the patients' families. The patients' records were reviewed retrospectively. Patients were identified according to acute myocarditis criteria: 1) recent history of viral infection with fever; 2) acute onset cardiac failure signs and/or cardiogenic shock; 3) abnormal ECG, increase in cardiac enzymes [creatinine kinaseMB (CK-MB), troponin-I); 4) left ventricular dysfunction signs in echocardiography [ejection fraction (EF) $<40 \%$, contractile function (CF) $<25 \%$ ]; and 5) no previously known cardiac disease. Patients who met the criteria were diagnosed as having acute myocarditis. Of these, patients with a severe progressive condition with no response to medical treatment were evaluated as having AFM.

Demographic information, clinical and vital signs, laboratory results [leukocytes, C-reactive protein (CRP), CK, CK-MB, troponin-I, pro-BNP, urea, creatinine, aspartate aminotransferase (AST), alanine aminotransferase (ALT)], echocardiography measurements, detectable viral-bacterial causes, pre- and post-ECMO vasoactive inotropic score (VIS), Pediatric Risk of Mortality (PRISM) score, ventilation parameters, and renal replacement treatment and duration were recorded.

All patients received VA ECMO. Maquet or Medos systems were used. Catheterization was performed through cardiovascular surgery. In two patients, right femoral arteries and right femoral veins were used, in others, right common carotid artery and right internal jugular veins were used. A perfusionist was present 24 hours per day in our hospital. Before catheterization, patients received heparin at a loading dose of $50 \mathrm{U} / \mathrm{kg}$. During ECMO, heparin infusion continued by following the activated clotting time (ACT). Keeping ACT between 170-220 sec, hemoglobin levels $>10 \mathrm{~g} / \mathrm{dl}$, thrombocyte number $>100,000 /$ $\mathrm{mm}^{3}$ was targeted. Adjunct flow volume was adjusted according to the hemodynamic insides and was maintained at 100-150 ml/ $\mathrm{kg} / \mathrm{min}$ to keep venous oxygen saturation at $>70 \%$. The $\mathrm{FiO}_{2}$ (fraction of inspired oxygen) of the air and oxygen mixture entering the membrane lung was kept at $40-60 \%$ and arterial oxygen saturation at $>94 \%$. Patients were evaluated through daily echocardiographic and chest X-rays. All patients received pressure-controlled synchronized intermittent mechanical ventilation. Throughout ECMO, the following parameters were kept between subsequent values: $\mathrm{FiO}_{2} 30-45 \%$, frequency 10-18/min, PEEP (positive end-expiratory pressure) 8-12 $\mathrm{cmH}_{2} \mathrm{O}$. All patients received continuous renal replacement therapy on the ECMO system.

ECMO flow rates were decreased when sufficient improvement of cardiac functions were seen in patients examined by transthoracic echocardiography, and the heparin dose to prevent thrombosis and vasoactive-inotropic drugs were increased. ECMO was terminated after observing sufficient cardiac functions upon reducing ECMO flow rate by $50 \%$ and patients were decannulated.

\section{Results}

Three male and 3 female patients with a mean age of 63 months were included in the study. The mean ECMO treatment duration was 164 hours, and mean length of intensive care stay was 24 days. The demographic features of the patients are summarized in Table I. The most common symptoms were chest pain $(66 \%)$, fever $(66 \%)$, and cough $(50 \%)$. All of the patients had severe circulatory failure and severe arrhythmia [supraventricular tachycardia (SVT), pulseless ventricular tachycardia (VT)] seen in two 
patients. Patients with severe arrhythmia were unresponsive to anti-arrhythmic treatment, and cardiac contractility dysfunction with arrhythmia caused severe circulatory collapse. There was no response to cardioversion and defibrillation. After ECMO support, rhythmic irregularity seemed to be under control in these patients. One patient received extracorporeal cardiopulmonary resuscitation (ECPR). One patient was followed as having juvenile idiopathic arthritis (JIA), one patient had Becker muscular dystrophy (BMD), and one patient as having ventricular septal defect (VSD). The remaining patients were previously known to be healthy. The cardiac functions of the patient with BMD were completely normal in echocardiography performed 3 months earlier on intensive care admission. After presenting with severe cardiogenic shock following an upper respiratory tract infection, the patient was accepted as having AFM, which developed despite having stable cardiac function in the follow-up. Eighty three percent (n: 5) of patients were weaned successfully from ECMO. Cardiac functions were fully recovered in the first and sixth-month followups of the decannulated patients; all were discharged without neurologic sequelae. The survival rate was $83 \%$. Due to a lack of adequate improvement in cardiac functions on the 30th day of internalization, the patient with BMD was transferred to another center where they could perform implantation of a ventricular support system under ECMO and be included on a heart transplantation list. Unfortunately, the patient died of multiple organ failure on the 16th day of hospitalization in the referral center.

All patients were supported with high doses of vasoactive drugs prior to ECMO (milrinone $0.75 \mathrm{mcg} / \mathrm{kg} / \mathrm{min}$, noradrenaline $0.6-1.5 \mathrm{mcg} /$ $\mathrm{kg} / \mathrm{min}$, adrenaline $0.6-1.2 \mathrm{mcg} / \mathrm{kg} / \mathrm{min}$, dobutamine $10-15 \mathrm{mcg} / \mathrm{kg} / \mathrm{min}$, dopamine 10$15 \mathrm{mcg} / \mathrm{kg} / \mathrm{min})$. Before ECMO, the patients' mean vasoactive inotropic score (VIS) was 170 , the mean PRISM score was 25.2, and the mean pediatric logistic organ dysfunction score (PELOD) was 29.2. After ECMO, milrinone treatment continued $(0.25-0.5 \mathrm{mcg} / \mathrm{kg} / \mathrm{min})$. Patients were evaluated using transthoracic echocardiography before ECMO and the mean left ventricular ejection fraction was $18 \%$.
All patients were on ECMO life support within 2 hours of the indication being recognized. Heart rate (HR), systolic blood pressure, blood gas $[\mathrm{pH}$, partial pressure of carbon dioxide $\left(\mathrm{pCO}_{2}\right)$, arterial partial pressure of oxygen $\left.\left(\mathrm{paO}_{2}\right)\right]$, oxygen saturation, and lactate levels before ECMO and 1st hour of ECMO were compared. After ECMO support, heart rate, blood pressure, and oxygen saturation in all patients improved greatly (Table I and II).

During ECMO, 1 patient had nasopharyngeal hemorrhage, 1 patient had hematuria, and one patient's cannula required changing because it was perforated. Nasopharyngeal bleeding stopped with tamponade. All patients received continuous veno-venous hemodiafiltration (due to inadequate end-organ perfusion and acute renal injury) treatment combined with an ECMO circuit.

\section{Discussion}

Acute fulminant myocarditis is a relatively uncommon clinical condition in children, but the prevalence in critically ill pediatric patients is still unknown. In our two-year study period, 952 patients were internalized to our PICU, and 22 patients in total were diagnosed as having acute myocarditis. Seventy-two percent of these patients were responsive to medical treatment, but six patients were unresponsive to medical treatment and needed ECMO support for AFM.

Despite much research on acute myocarditis, it remains difficult to diagnose and evaluate in childhood. Especially in younger children, nonspecific signs and symptoms make it harder to diagnose. Clinical observation is diagnostic, and some laboratory findings, chest X-ray, electrocardiogram (ECG), and echocardiography can be helpful. In our study, the most common symptoms were fever, chest pain, and cough. In other studies, the most common symptoms were respiratory distress, fever, gastrointestinal symptoms, chest pain, and restlessness. ${ }^{8,10}$

The mortality rate in AFM is above $50 \%$ if not supported by ECMO. ${ }^{11,12}$ In our study, five of our patients survived $(83 \%)$. This result is above the average survival rate according to several studies. Most important reasons for 


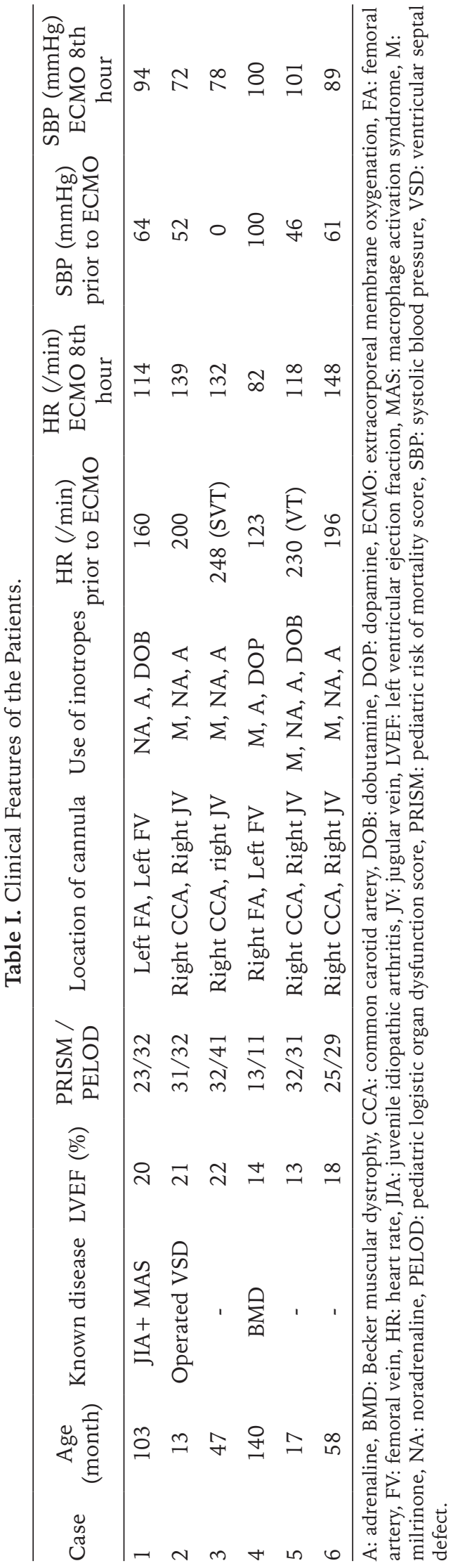

our higher survival rate are early diagnosis and short ECMO support starting duration. Delay in diagnosis and treatment leads to an increase in morbidity and mortality. ${ }^{13}$ In our study, the time between identifying the ECMO indication and treatment initiation was less than 2 hours. ECMO treatment was initiated in the first 24 hours of intensive care internalization of all patients. In a study including 17 patients with myocarditis by Jung et al. ${ }^{14}, 13$ patients had extracorporeal life support, 4 patient had ECMO in 24 hours after admission, three of whom died. In another study that reviewed patients with AFM, it was observed that $75 \%$ of patients who do not survive had ECMO support 24 hours after admission. ${ }^{12}$ This shows the starting ECMO support early results in better outcomes because it parallels with our study.

Reviewing previous studies, the most common ECMO indications are severe arrhythmia, end-organ failure, and circulation failure. ${ }^{15,16}$ Clinical arrhythmia alone does not require ECMO; however, when combined with circulation failure and end-organ failure in the same patient, ECMO is needed. Two of our patients had severe arrhythmia, one of whom had pulseless VT secondary to myocarditis. There was no response to antiarrhythmic treatment and defibrillation, after ECPR the patient's circulatory failure and dysrhythmia recovered. The other patient was admitted from another center to our PICU with supraventricular tachycardia refractory to medical treatment. When the patient's family history was investigated, it was found that several family members had a history of arrhythmia and heart disease. ECMO was initiated for the patient who had circulatory failure and cardiogenic shock. Arrhythmia continued for 24 hours under ECMO, after 24 hours the dysrhythmia was under control with medical treatment.

$\mathrm{Wu}$ et al. ${ }^{13}$ compared patients with acute myocarditis according to their need for ECMO in their study and found that female sex, arrhythmia, left ventricular ejection fraction (LVEF) $<60 \%$, high CK-MB and troponin I levels (>14.21 ng/ml) were significantly higher in the group needing ECMO. Although there was no significant difference in terms of sex due to the low patient number, troponin 


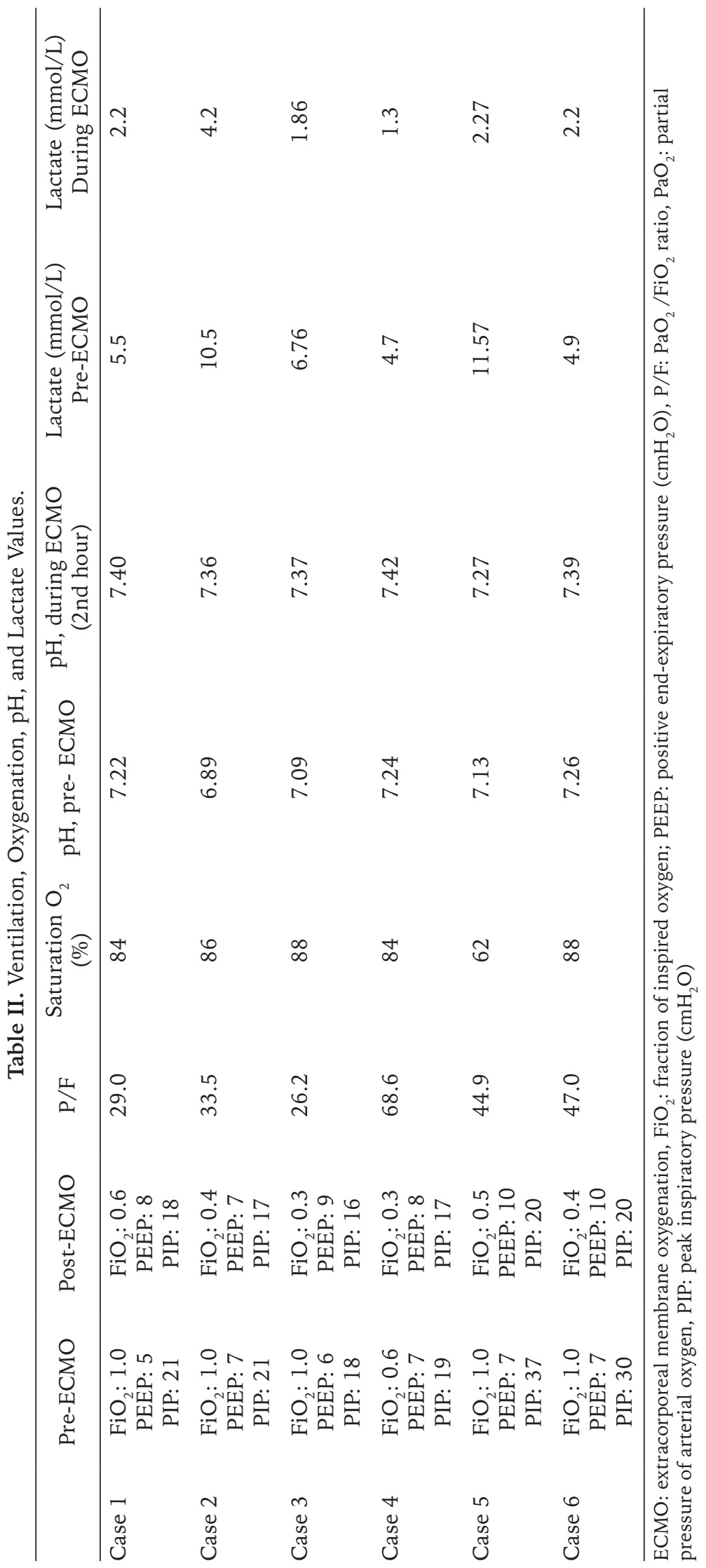


I, CK-MB and proBNP were found to be high. Furthermore, in all patients, LVEF was well below $60 \%$. Al-Biltagi et al. ${ }^{17}$ demonstrated a relationship between low LVEF levels and poor prognosis in patients with myocarditis. Lee et al. ${ }^{12}$ compared factors that affected mortality in patients with myocarditis and presented the following results; patients who survived had a mean LVEF of $30 \%$, whereas patients who did not survive had a mean LVEF of $19 \%$. Our patients had a mean of LVEF of $18 \%$ at the time of admission, the non-survivor patient's LVEF was $14 \%$. However, no statistical significance was detected because the patient number was small.

There were some limitations to the study. First, it was a single-center study evaluating a small number of patients retrospectively. Second, although having performed a respiratory viral panel in all patients, accurate diagnosis of the viral agent causing myocarditis was not achieved nor were endomyocardial biopsies taken.

In conclusion, diagnosing acute myocarditis in childhood is challenging, but suspicious laboratory results and echocardiography can be helpful to support the diagnosis. Irregular rhythms must be checked in all patients who present with cardiogenic shock symptoms. Symptomatic patients with abnormal ECG findings must be evaluated for myocarditis. Early ECMO support is life-saving for patients who do not respond to inotropic treatment and respiratory support, in patients developing cardiogenic shock, and end-organ failure due to refractory arrhythmias.

\section{REFERENCES}

1. Kearney MT, Cotton JM, Richardson PR, Shah AM. Viral myocarditis and dilated cardiomyopathy: Mechanisms, manifestations and management. Postgrad Med J 2001; 77: 4-10.

2. Cooper LT Jr. Myocarditis. N Engl J Med 2009; 360: 1526-1538.

3. Durani Y, Egan M, Baffa J, Selbst SM, Nager AL. Pediatric myocarditis: presenting clinical characteristics. Am J Emerg Med 2009; 27: 942-947.

4. Brown $\mathrm{KL}$, Ichord $\mathrm{R}$, Marino $\mathrm{BS}$, Thiagarajan RR. Outcomes following extracorporeal membrane oxygenation in children with cardiac disease. Pediatr Crit Care Med 2013; 14: 73-83.
5. Wilmo I, Morales DL, Price JF, et al. Effectiveness of mechanical circulatory support in children with acute fulminant and persistent myocarditis. J Card Fail 2011; 17: 487-494.

6. Teele SA, Allan CK, Laussen PC, Newburger JW, Gauvreau K, Thiagarajan RR. Management and outcomes in pediatric patients presenting with acute fulminant myocarditis. J Pediatr 2011; 158: 638-643. e1.

7. Rajagopal SK, Almond CS, Laussen PC, Rycus PT, Wypij D, Thiagarajan RR. Extracorporeal membrane oxygenation for the support of infants, children, and young adults with acute myocarditis: a review of the Extracorporeal Life Support Organization registry. Crit Care Med 2010; 38: 382-387.

8. Shu-Ling C, Bautista D, Kit CC, Su-Yin AA. Diagnostic evaluation of pediatric myocarditis in the emergency department: a 10-year case series in the Asian population. Pediatr Emerg Care 2013; 29: 346351.

9. Freedman SB, Haladyn JK, Floh A, Kirsh JA, Taylor G, Thull-Freedman J. Pediatric myocarditis: emergency department clinical findings and diagnostic evaluation. Pediatrics 2007; 120: 1278-1285.

10. Vashist S, Singh GK. Acute myocarditis in children: current concepts and management. Curr Treat Options Cardiovasc Med 2009; 11: 383-391.

11. Kodama M, Oda H, Okabe M, Aizawa Y, Izumi T. Early and long-term mortality of the clinical subtypes of myocarditis. Jpn Circ J 2001; 65: 961-964.

12. Lee EY, Lee HL, Kim HT, Lee HD, Park JA. Clinical features and short-term outcomes of pediatric acute fulminant myocarditis in a single center. Korean J Pediatr 2014; 57: 489-495.

13. Wu HP, Lin MJ, Yang WC, Wu KH, Chen CY. Predictors of extracorporeal membrane oxygenation support for children with acute myocarditis. Biomed Res Int 2017; 2017: 2510695.

14. Jung SY, Shin HJ, Jung JW, et al. Extracorporeal life support can be a first-line treatment in children with acute fulminant myocarditis. Interact Cardiovasc Thorac Surg 2016; 23: 247-252.

15. Duncan BW, Bohn DJ, Atz AM, French JW, Laussen PC, Wessel DL. Mechanical circulatory support for the treatment of children with acute fulminant myocarditis. J Thorac Cardiovasc Surg 2001; 122: 440-448.

16. Kato S, Morimoto S, Hiramitsu S, et al. Risk factors for patients developing a fulminant course with acute myocarditis. Circ J 2004; 68: 734-739.

17. Al-Biltagi M, Isaa M, Hagar HA, Abdel-Hafez M, Aziz NA. Circulating cardiac troponins levels and cardiac dysfunction in children with acute and fulminant viral myocarditis. myocarditis. Acta Paediatr 2010; 99: 1510-1516. 